\title{
Mergel, Thomas: Köln im Kaiserreich. 1871-1918 (Geschichte der Stadt Köln, Bd. 10), 584 S., Greven, Köln 2018.
}

\section{Dieter Schott}

Online publiziert: 12. Mai 2020

(C) Der/die Autor(en) 2020

Thomas Mergels monumentale Stadtgeschichte Kölns im Kaiserreich, vom GrevenVerlag hervorragend ausgestattet - die zahlreichen historischen Abbildungen weisen eine selten gesehene Qualität auf - wird sowohl unter den Nicht-Kölner Stadthistorikern als auch im an ,Colonia“ interessierten lokalen und regionalen Publikum eine dankbare Leserschaft finden. Denn die in Mergels programmatischer Einleitung aufgewiesene Dichotomie eines solchen Werkes, entweder eine die Eigenheit betonende Stadtbiografie zu schreiben, oder - im vergleichend urbanisierungsgeschichtlichen Zugriff - die Stadt ,als ein Beispiel für eine Moderne [zu beschreiben], die sich so oder ähnlich auch anderswo zugetragen hat" (S. 1), wird bewusst vermieden. Mergel möchte beides tun, was er mit der Kölner Modernisierung begründet: „Die Betonung des Besonderen, die sich in Köln finden lässt, wohl ausgeprägter als in vielen anderen Städten, ist in hohem Maße eine Begleiterscheinung, wenn nicht sogar eine Frucht des Wegs der Stadt in die urbane Moderne“" (S. 2). Den konzeptionellen Anspruch einer histoire totale, den der Autor ebenfalls in der Einleitung erhebt, kann die Stadtgeschichte über weite Strecken recht überzeugend einlösen, denn Mergel spürt auch dem nach, was die Großprozesse der Urbanisierung und Industrialisierung für das Leben der einzelnen Städter bedeutet haben, wie sie erfahren, erlebt und erlitten wurden. Mergel fragt, neben der Nachzeichnung der administrativ-technischen Entwicklung der Stadt, der Stadt als Ort politischer Kultur und als Sozialkörper, auch nach der Erfahrungsdimension der Einzelnen, die nach Köln gewandert sind, sich dort erfolgreich etabliert haben oder wieder weggezogen sind, nach den unglaublich hohen Mobilitätsraten in der Stadt, der Schule, dem Gesund- oder Kranksein, der Kriminalität oder der Gewaltneigung in der Bevölkerung. Er nutzt dabei neben Sekundärliteratur vor allem zeitgenössische Quellen, insbesondere die städtische Statistik, die detaillierten Verwaltungsberichte der Stadt, aber auch Ego-Dokumente und Beobachtungen in den Medien, Berichte der staatlichen Stellen, die regelmäBig feinkörnige Bestandsaufnahmen der politischen, sozialen, wirtschaftlichen und kulturellen Gesamtsituation Kölns an die Berliner Regierungsbürokratie lieferten. In Anbetracht des spektakulären Zusammenbruchs des Kölner Stadtarchivs 2009 macht Mergel gewissermaßen aus der Not eine Tugend: Er wich teilweise auf an-

D. Schott $(\bowtie)$

Technische Universität Darmstadt, Darmstadt, Deutschland

E-Mail: schott@pg.tu-darmstadt.de 
dere Archive aus, in denen die staatliche Überlieferung des Blicks der preußischen Bürokratie auf Köln aufbewahrt wurde, nutzte Wirtschaftsarchive und das umfangreiche zeitgenössische Schrifttum. Seine Perspektive ist die Rekonstruktion des Erfahrungswandels. Er will den „Prozess des Lernens“ in der Auseinandersetzung der Menschen mit der sich rapide wandelnden Großstadt nachzeichnen.

Das Buch gliedert sich in vier Großthemen und insgesamt 21 Kapitel. Zunächst stellt Mergel unter „Eine rheinische Stadt im neuen Reich“ Köln in der neuen politischen Landschaft des Kaiserreichs in den 1870er und frühen 1880er Jahren vor, präsentiert das Verhältnis von Stadt und Staat, die Struktur der Stadtverwaltung, ihre Oberbürgermeister, geht auf die politischen Lager und ihre Kämpfe ein. Besondere Aufmerksamkeit gilt dem Kulturkampf der 1870er Jahre, der in einer so ausgeprägt katholischen Stadt wie Köln besonders hohe Wogen schlug. Allerdings unterstreicht Mergel auch, dass bei aller Schärfe der Konflikte im Zeichen neuer politischer Konstellationen sowohl die katholischen Repräsentanten vor Ort wie auch die liberalen Protagonisten bestrebt waren, den Konflikt wieder zu befrieden und einzuhegen.

Das zweite Großthema „Die Stadt in der Erweiterung“ setzt an dem für Köln zentralen Umstand an, dass die Stadt bis 1881 noch von der mittelalterlichen Stadtmauer und einem anschließenden Festungsgürtel eingeschnürt war und daher ein sehr viel langsameres Wachstum erlebte als Nachbarstädte oder die sich industrialisierenden Vororte. Mit der Niederlegung der Mauer, der Eingemeindung des Festungsgürtels 1883 und der ,großen Eingemeindung“von 1888, als die Vororte wie Bayenthal, Ehrenfeld und Nippes, aber auch das rechtsrheinische Deutz Teil der Großstadt Köln wurden, war daher ein Befreiungsschlag verbunden, der Köln den Entwicklungsrückstand innerhalb weniger Jahre aufholen ließ. Mergel zeichnet den Aufstieg des Munizipalsozialismus in dieser Periode nach, zeigt, wie die Stadtverordneten ihre anfängliche Zurückhaltung gegenüber kommunalem wirtschaftlichem Engagement aufgaben und verdeutlicht, wie sich Köln von der walking city zu einem technisch integrierten und differenzierten Verkehrs- und Kommunikationsraum entwickelte.

Im dritten Großthema „Die rheinische Metropole“ beleuchtet Mergel wirtschaftliche Veränderungsprozesse, Verhältnisse am Arbeitsplatz, soziale Hierarchien, Klassenverhältnisse wie auch den Umgang mit sozialen Disparitäten. Im Zuge der raschen Industrialisierung, ,proletarisierte“ sich die Bürgerstadt schnell, wobei die politischen Vertreter der Arbeiterschaft, die Sozialdemokraten, nur langsam und mühsam in Köln Fuß fassen konnten. Die ,Erfahrungsgeschichte" ist insbesondere in Kapitel 11 „Leben in der Großstadt“ vertreten, wo Mergel vom „Aufwachsen in der Großstadt“" über Orte der Geselligkeit, Wohnverhältnisse, Kriminalität und Prostitution ein breites Panorama der Alltagsgeschichte Kölns um 1900 zeichnet. Er geht dabei häufig von zeitgenössischen Stereotypen und Problematisierungen aus, etwa dem Bild der ,Großstadtnomaden ‘ aufgrund extremer Mobilitätsraten, und versucht klarzumachen, inwieweit diese zeitgenössischen Wahrnehmungen ideologisch eingefärbt waren. Zuwanderer migrierten meist nicht in eine vollständig unbekannte Umwelt, sondern hatten Anlaufpunkte und Orientierungsfaktoren. Exemplarisch verdeutlicht Mergel dies im Mikro-Maßstab an zwei sozialstrukturell sehr unterschiedlichen Straßen, deren Sozialprofil er herausarbeiten, zugleich aber auch zeigen kann, dass bei aller Fluktuation der Haushaltsvorstände das häufige Vorkommen bestimmter Nachnamen auf Kettenwanderungen in Verwandtschaftsnetzwerken hinweist. Ein 
wunderbares Beispiel, wie sich Modernisierung im Alltag, etwa in der Sterbekultur auswirkte, ist das 1910 gehaltene Plädoyer des Oberbürgermeisters Max Wallraf dafür, den traditionellen Leichenzug, der bislang vom Haus des Verstorbenen zum Friedhof ging, auf den Friedhof selbst zu verlegen: „Aber wenn der Trauerkondukt sich aus dem Trauerhause herausbewegt auf die Straße, sich durchwindet durch den großstädtischen Verkehr, durch die rasselnden Wagen, die klingenden Elektrischen, die sausenden Automobile - wer da nicht zu den allernächsten Leidtragenden gehört, den wird die Alltagsumgebung sehr bald wieder in die Alltagsstimmung zurückdrängen“ (S. 298). Sehr stark sind auch Passagen wie die, in der Mergel in der Erklärung der hohen Kriminalitätsziffern plausibel macht, dass dies gar nicht primär Resultat der Großstadtwerdung Kölns darstellt, sondern auf ländliche Delinquenz zurückgeht, die sich nunmehr, angesichts großstädtischer polizeilicher Ermittlungsintensität, in gesteigerten Kriminalitätsraten niederschlug. Bemerkenswert findet Mergel das trotz lautstarker Wahlkämpfe vergleichsweise konsensuelle Miteinander in der lokalen Politik, insbesondere zwischen Zentrum und Liberalen, was er auf die starke Stellung des Zentrums zurückführt, das bereits 1903 mit der zweiten Wählerklasse auch die Mehrheit in der Stadtverordnetenversammlung eroberte, aber eine im Reichsmaßstab eher liberale, deutlich sozialpolitisch akzentuierte Richtung vertrat. Zugleich wird dies auch einer kölnischen Mentalität des ,Leben und leben lassen“ zugeschrieben, die im Unterschied zu stärker antagonistischen Konfrontationen etwa in den Ruhrgebietsstädten oder in Berlin Konflikte einhegte und beherrschbar machte.

Die zunächst von diesem Konsens noch ausgeschlossenen Sozialdemokraten reihten sich im vierten Großthema des Buchs, „Der Erste Weltkrieg“, ebenfalls in die Burgfriedensgemeinschaft ein und vertraten bis in den Sommer 1918, im Unterschied zur SPD anderswo, ungebrochen die Kriegspolitik. Im Weltkrieg wurde das Sonderbewusstsein der Stadt Köln noch deutlicher artikuliert, als Konrad Adenauer, erster Beigeordneter und ab 1917 Oberbürgermeister der Stadt, bestrebt war, die Versorgung Kölns stärker auf lokale Ressourcen als auf die Einbeziehung in die hochbürokratischen kriegswirtschaftlichen Versorgungssysteme zu gründen.

Im knappen Fazit „Die Großstadt lernen“ unterstreicht Mergel die erstaunliche Integrationsfähigkeit der sich rasch wandelnden Großstadt, die den Neubürgern vielfache Möglichkeiten bot, sich im „Veedel“ (Viertel), im Verein, im Karneval oder der Kirchengemeinde heimisch zu machen. Auch die Pflege der baulichen Relikte des alten Köln, die starke Rolle des Doms als Identifikationssymbol oder des Kölner Brauchtums offenbart Mergel als Produkte der rapiden Urbanisierung des Kaiserreichs.

Die besondere Stärke von Mergels eindrucksvoller Geschichte Kölns liegt in erster Linie in der erfahrungsgeschichtlichen Durchdringung des Urbanisierungsprozesses. Sehr vielschichtig bringt der Autor zeitgenössische Beobachtungen und Problematisierungen zusammen mit Interpretationen der jüngeren sozial- und kulturgeschichtlichen Forschung etwa zu Migration, Gewalt oder Sexualität. Die Passagen zur Stadtgeschichte als Verwaltungsgeschichte zeigen zwar klar den Aufbau der Leistungsverwaltung, aber meist werden ,nur' die Ergebnisse der teilweise jahrelangen Planungs- und Beratungsprozesse präsentiert, der Weg zum Ziel, oder auch die dabei auftretenden Sackgassen und nicht verfolgten Alternativen werden kaum näher beleuchtet; sie wären für den komparativ interessierten Stadthistoriker ebenfalls 
von großem Interesse. Die großartige visuelle Ausstattung mit Bildern, die jeweils kundig kommentiert werden, hat leider auf der statistischen Ebene keine Entsprechung. Obwohl Mergel intensiv mit Zahlen arbeitet und argumentiert, findet sich im Band kaum eine Tabelle. Der an kurzer quantitativer Orientierung interessierte Leser bekommt demnach keinen Überblick der Einwohnerentwicklung der Gesamtstadt und der Stadtteile, der Entwicklung des Kommunalhaushalts und der Schulden, der Kriminalität oder der Mobilitätszahlen, was durch das detaillierte Sachregister nur unvollkommen kompensiert wird. Nichtsdestotrotz kann Thomas Mergels „Köln im Kaiserreich“ als Standard für eine erfahrungs- und kulturgeschichtlich orientierte Stadtbiografie gelten, die Köln zugleich in den Gesamtkontext der Urbanisierung einbettet.

Funding Open Access funding provided by Projekt DEAL.

Open Access Dieser Artikel wird unter der Creative Commons Namensnennung 4.0 International Lizenz veröffentlicht, welche die Nutzung, Vervielfältigung, Bearbeitung, Verbreitung und Wiedergabe in jeglichem Medium und Format erlaubt, sofern Sie den/die ursprünglichen Autor(en) und die Quelle ordnungsgemäß nennen, einen Link zur Creative Commons Lizenz beifügen und angeben, ob Änderungen vorgenommen wurden.

Die in diesem Artikel enthaltenen Bilder und sonstiges Drittmaterial unterliegen ebenfalls der genannten Creative Commons Lizenz, sofern sich aus der Abbildungslegende nichts anderes ergibt. Sofern das betreffende Material nicht unter der genannten Creative Commons Lizenz steht und die betreffende Handlung nicht nach gesetzlichen Vorschriften erlaubt ist, ist für die oben aufgeführten Weiterverwendungen des Materials die Einwilligung des jeweiligen Rechteinhabers einzuholen.

Weitere Details zur Lizenz entnehmen Sie bitte der Lizenzinformation auf http://creativecommons.org/ licenses/by/4.0/deed.de. 